\title{
$17 \beta$-Estradiol Protects Primary Macrophages Against HIV Infection Through Induction of Interferon-Alpha
}

\author{
Carley Tasker,, ${ }^{1, *}$ Jian Ding, ${ }^{2, *}$ Mirco Schmolke, ${ }^{3}$ Amariliz Rivera-Medina, ${ }^{4}$ \\ Adolfo García-Sastre, ${ }^{3}$ and Theresa L. Chang ${ }^{1,2}$
}

\begin{abstract}
Estrogen has been shown to increase resistance to HIV/SIV transmission by increasing the thickness of the genital epithelium. The immunological role of estrogen in HIV infection of primary target cells is less well characterized. We have found that primary macrophages are a target for anti-HIV activity of $17 \beta$-estradiol (E2). E2 did not affect surface expression of CD4 and HIV co-receptors nor HIV attachment to monocyte-derived macrophages (MDMs). In addition, E2 treatment blocked infection by a co-receptor-independent HIV-1 ${ }_{\mathrm{VSV}-\mathrm{G}}$ pseudotyped virus. Quantitative polymerase chain reaction analysis of HIV reverse transcribed DNA products indicated that E2 blocked HIV reverse transcription. E2 upregulated gene expression of interferons (IFNs) in MDMs from multiple donors. However, induction of host restriction factors APOBEC3G, APOBEC3F, or SAMHD1 was not consistent, with exception of APOBEC3A. Anti-HIV activity of E2 was abolished in the presence of IFN- $\alpha$ neutralizing antibody, and was absent in bone marrow-derived macrophages from IFN- $\alpha$ receptor deficient mice. Interestingly, HIV overcame E2-mediated HIV inhibition by suppressing induction of IFNs when MDMs were exposed to HIV before E2 treatment. These results offer a new mechanism of E2 on HIV inhibition. Future studies on the interplay between HIV and E2-mediated innate immune responses will likely provide insights relevant for development of effective strategies for HIV prevention.
\end{abstract}

\section{Introduction}

G LOBALLY, MORE THAN 150 MILLION WOMEN use hormone contraceptives, and 16 million of these women live in sub-Saharan Africa (21), where the HIV/AIDS epidemic has significant impact. The effect of hormone contraceptive use on HIV acquisition and transmission has profound implications for family planning policies in countries with high rates of HIV transmission. In rhesus macaques, estrogen protects against vaginal transmission of SIV (45), whereas progestins such as depot medroxyprogesterone acetate (DMPA; Depo-Provera) increase the risk of SIV/SHIV acquisition $(30,49)$. In humans, results of prospective studies on the role of hormonal contraceptive use in HIV acquisition and transmission have been inconsistent $(17-19,28,31,34,40,50)$, in part due to different methods of hormonal contraceptives, divergent biological activities of various progestins, and other confounding factors such as condom usage. Despite the controversy regarding the ef- fect of hormone contraceptive use on HIV acquisition and transmission, estrogen is thought to exhibit a protective effect against HIV/SIV transmission (reviewed in (19)).

Studies in macaques and humans have suggested that estrogen protects the host against HIV infection through enhancement of physical barriers at the genital mucosa by increasing the thickness of the genital epithelium. Estrogen induces thickening of the vaginal epithelium in postmenopausal women on hormone replacement therapy $(14,33)$ and in ovariectomized macaques $(44,45)$. Additionally, in humans, it also rapidly keratinizes the inner foreskin, the site for HIV entry in the penis (38), and may protect men against HIV. Postmenopausal women who have lower estrogen and thinner vaginal epithelium (36) are more susceptible to HIV transmission (European Study Group on Heterosexual Transmission of HIV, 1992).

In contrast to its effect on the genital epithelium, the immunological role of estrogen in HIV protection is understudied. Estrogen exerts both inflammatory and anti-

This work was presented at the 20th Conference on Retroviruses and Opportunistic Infections (CROI) in Atlanta, Georgia, May 2-6, 2013 (Abstract \#240).

Departments of ${ }^{1}$ Microbiology and Molecular Genetics and ${ }^{4}$ Medicine and ${ }^{2}$ Public Health Research Institute, Rutgers Biomedical and Health Sciences-New Jersey Medical School, Newark, New Jersey.

${ }^{3}$ Department of Microbiology, Mount Sinai School of Medicine, New York, New York.

*Authors contributed equally to this work. 
inflammatory effects depending on various factors such as target cells, immune stimuli, microenvironment, and estrogen concentration (reviewed in (46)). Enhanced HIV replication has been found in ex vivo ectocervical tissue from postmenopausal women compared to tissue from premenopausal women, and this HIV enhancement is associated with increased inflammation (42). However, the effect of estrogen on HIV infection of specific primary target cells and its underlying mechanism has not been well defined.

Macrophages are one of the major HIV target cells present in the female genital mucosa (43). While estrogen generally suppresses production of pro-inflammatory cytokines including IL-6, IL- $1 \beta$, and TNF- $\alpha$ by monocytes and macrophages $(15,24,32)$, it promotes IFN- $\gamma$ production (23) and TLR4-mediated pro-inflammatory cytokine production through activation of macrophages (4). In this study, we found that $17 \beta$-estradiol (E2) protected primary macrophage against HIV infection, and we demonstrate a novel mechanism of E2mediated HIV inhibition through IFN- $\alpha$ induction.

\section{Materials and Methods}

\section{Reagents}

Recombinant human IL-2, mouse anti-human CD3 Ab (clone UCHT1), and mouse anti-human CD28 Ab (clone 37407.111) were purchased from R\&D Systems (Minneapolis, MN). Mouse anti-human IFN- $\alpha$ mAb (clone MMHA2) was obtained from PBL Interferon Source (Piscataway, NJ). Water-soluble E2, water-soluble progesterone (P4), Histopaque $^{\circledR}-1077$, Triton X-100, RPMI-1640 medium, fetal bovine serum (FBS), modified RPMI-1640 medium (phenol red free), charcoal-treated FBS, human AB serum, and phytohemagglutinin (PHA) were purchased from Sigma-Aldrich (St. Louis, MO). PerCP-conjugated mouse anti-human CD4 (clone RPA-T4) was purchased from Biolegend (San Diego, CA). APC-conjugated mouse anti-human CD184 (CXCR4, clone 12G5), APC-conjugated mouse anti-human CD195 (CCR5, clone 3A9), and mouse IgG1 and IgG2a isotype controls were purchased from BD Biosciences (San Jose, CA).

\section{Cell isolation and culture}

All primary cells were cultured with RPMI-1640 medium supplemented with $10 \%$ heat-inactivated FBS. Peripheral blood mononuclear cells (PBMCs) from normal healthy blood donors were isolated by Histopaque ${ }^{\circledR}-1077$ gradient centrifugation. Monocytes were isolated from PBMCs by adherence in flasks coated with human AB serum. Adherent cells were maintained in Roswell Park Memorial Institute medium (RPMI) with 20\% FBS for 7 days and allowed to differentiate into monocyte derived macrophages (MDMs). FACS analysis showed that more than $95 \%$ of cells were CD68 +, a marker for differentiated macrophages. CD4 + T-cells were isolated from PBMCs or peripheral blood lymphocytes (PBLs) by negative selection using a $\mathrm{CD} 4+\mathrm{T}$-cell isolation kit II (Miltenyi, Auburn, CA). PBMCs or CD4+ T-cells were then activated by incubation with PHA $(5 \mu \mathrm{g} / \mathrm{mL})$ and IL-2 (50 IU/mL) or by immobilized anti-CD3 Ab $(1 \mu \mathrm{g} / \mathrm{mL}) /$ anti-CD28 Ab $(0.6 \mu \mathrm{g} / \mathrm{mL})$ for 3 days. Activated cells were washed with PBS and incubated with IL-2 before use.
Before hormone treatment, all primary cells were incubated for $24 \mathrm{~h}$ in phenol red-free RPMI-1640 containing $10 \%$ heat-inactivated, charcoal-treated FBS.

\section{HIV-1 infection}

Replication-defective HIV-1 JR-FL or HIV-1 ${ }_{\text {vsv }}$ Envpseudotyped luciferase expressing reporter viruses used in single-cycle infection assays were produced in HEK293Tcells by co-transfection of a plasmid encoding the envelope deficient HIV NL4-3 virus and luciferase reporter gene (pNL-Luc-R+E-; gift of N. Landau, New York University, New York, NY) along with a plasmid encoding HIV-1 $1_{\text {JR-FL }}$ or VSV G envelope as described previously $(6,8)$. Cells (PBMC or CD4 + T-cells at $1 \times 10^{6}$ per sample or MDMs at $1 \times 10^{5}$ per sample) were infected with pseudotyped luciferase reporter viruses (8-10 ng HIV p24 per sample) for $2 \mathrm{~h}$ at $37^{\circ} \mathrm{C}$. After washing off unbound virus, MDMs or PBMCs/CD4 + T-cells were cultured for 2 or 3 days, respectively, before lysis in passive lysis buffer (Promega, Madison, WI). Luciferase activity (in relative light units) was measured on a Glomax 20/20 luminometer (Promega).

For multiple-round infection assays, cells were infected with CCR5-using HIV-1 $1_{\mathrm{BaL}}$ virus (Advanced Biotechnologies, Inc., Columbia, MD) or primary isolates (the UNAIDS Network for HIV isolation and Characterization, Division of AIDS, National Institute of Allergy and Infectious Diseases) at a multiplicity of infection (MOI) of 0.05 for $2 \mathrm{~h}$. After washing off unbound virus, cells were cultured, and HIV-1 p24 levels in cell culture supernatant were measured at different time points after viral infection by enzyme-linked immunosorbant assay (ELISA; HIV-1 p24 ELISA kit; SAICFrederick, Inc., Frederick, MD).

\section{MTS assay}

MDMs were plated in 96-well plates at 10,000 cells per well. The cells were treated with various concentrations of E2 for $24 \mathrm{~h}$. Substrate from the CellTiter $96^{\circledR}$ Aqueous One Solution Cell Proliferation Assay (Promega) was added per the manufacturer's instruction. The optical density was measured at $490 \mathrm{~nm}$.

\section{FACS analysis}

MDMs were incubated at $4{ }^{\circ} \mathrm{C}$ in ice-cold PBS for $30 \mathrm{~min}$ and then detached using cell scrapers. Surface expression of CD4, CXCR4, and CCR5 were determined by staining the cells with fluorochrome-conjugated specific Abs. Appropriate isotype controls were included in all assays. Stained samples were analyzed on a BD Accuri ${ }^{\mathrm{TM}}$ C6 flow cytometer. Results were analyzed with FlowJo (Tree Star, Inc., Ashland, OR).

\section{Real-time reverse transcription polymerase chain reaction}

Total RNA was isolated from cells using TRIzol ${ }^{\circledR}$ (Life Technologies, Carlsbad, CA). To synthesize first-strand cDNA, $1000 \mathrm{ng}$ of total RNA, oligo $\mathrm{d}(\mathrm{T})_{16}(25 \mu \mathrm{g} / \mathrm{mL})$ and $\mathrm{dNTP}(0.5 \mathrm{mM})$ were incubated at $65^{\circ} \mathrm{C}$ for $5 \mathrm{~min}$ and quickchilled on ice. RT was performed at $42^{\circ} \mathrm{C}$ for $50 \mathrm{~min}$ using SuperScriptTM II (Life Technologies). The PCR contained 
cDNA equivalent to $30 \mathrm{ng}$ of RNA input, $200 \mathrm{nM}$ primer sets, and SYBR Green Master Mix (Qiagen, Valencia, CA), and was run in a StepOnePlus real-time PCR system (Invitrogen/Life Technologies). Each reaction was performed in triplicate. The primer sequences were: GAPDH forward (5'-AGG TGA CAC TAT AGA ATA CTC TCT GCT CCT CCT GTT CG-3'), GAPDH reverse (5'-GTA CGA CTC ACT ATA GGG AAC GAC CAA ATC CGT TGA CTC$\left.3^{\prime}\right)$; IFN- $\alpha$ forward $\left(5^{\prime}\right.$-CAC ACA GGC TTC CAG GCA TTC-3'), IFN- $\alpha$ reverse (5'-TCT TCA GCA CAA AGG ACT CAT CTG-3'); IFN- $\beta$ forward (5'-GAG CTA CAA CTT GCT TGG ATT CC-3'), IFN- $\beta$ reverse (5'-CAA GCC TCC CAT TCA ATT GC- $3^{\prime}$ ); IFN- $\gamma$ forward $\left(5^{\prime}\right.$-AGC TCT GCA TCG TTT TGG GTT-3'), IFN- $\gamma$ reverse (5'-GTT CCA TTA TCC GCT ACA TCT GAA-3'); APOBEC3A forward (5'-TGG CAT TGG AAG GCA TAA GAC-3'), APOBE C3A reverse $\left(5^{\prime}\right.$-TTA GCC TGG TTG TGT AGA AAG C$\left.3^{\prime}\right)$; APOBEC $3 \mathrm{~F}$ forward (5'-TAC GCA AAG CCT ATG GTC GG-3'), APOBEC3F reverse (5'-GCT CCA AGA TGT GTA CCA GG-3'); APOBEC3G forward (5'-GGC TCC ACA TAA ACA CGG TTT C-3'), APOBEC3G reverse (5'AAG GGA ATC ACG TCC AGG AA-3'); SAMHD1 forward (5'-CCA AGC GTC CCC GTT GCG AT-3'), SAMHD1 reverse $\left(5^{\prime}\right.$-TCA AAG CCA CCG CGC CTG AG-3'). PCR conditions included a $95^{\circ} \mathrm{C}$ denaturation for $10 \mathrm{~min}$ followed by 40 cycles of $95^{\circ} \mathrm{C}$ for $15 \mathrm{~s}$ and $56^{\circ} \mathrm{C}$ for $60 \mathrm{~s}$. Quantification of PCR products was normalized according to the amount of cDNA by amplifying GAPDH. Relative quantification of gene expression was calculated by using a $\Delta \Delta \mathrm{Ct}(\mathrm{Ct}$, threshold cycle of real-time PCR) method based on signal intensity of the PCR according to the following formula: $\Delta \mathrm{CT}=\mathrm{Ct}_{18 \mathrm{~S}}$ rRNA $-\mathrm{Ct}_{\text {target }}, \Delta \Delta \mathrm{Ct}=$ $\Delta \mathrm{Ct}_{\mathrm{control}}-\Delta \mathrm{Ct}_{\mathrm{E} 2}$, Ratio $=2^{-\Delta \Delta \mathrm{Ct}}$.

\section{Quantitative real-time PCR analysis of HIV-1 DNA}

Total DNA was extracted from HIV-infected MDMs using the QIAamp DNA Blood Mini Kit (Qiagen). The level of HIV RT products was determined by quantitative real-time PCR analysis. Each PCR reaction contained $100 \mathrm{ng}$ total DNA, primers (200 nM each), and SYBR Green Master Mix (Qiagen). The primer sequences for HIV-1 early RT products were M667 (5'-GGC TAA CTA GGG AAC CCA CTG-3') and AA55 (5'-CTG CTA GAG ATT TTC CAC ACT GAC$\left.3^{\prime}\right)$; the primers for HIV-1 late RT products were M667 and M661 (5'-CCT GCC TCG AGA GAG CTC CAC ACT GAC$\left.3^{\prime}\right)$ (53). Standard curve for early or late RT products was generated with 10-fold serial dilutions of pNL4-3.Luc.R-Eranging from $10^{1}$ to $10^{8}$ copies. PCR cycling conditions included $95^{\circ} \mathrm{C}$ for $10 \mathrm{~min}$, followed by 40 cycles of $95^{\circ} \mathrm{C}$ for $30 \mathrm{~s}, 55^{\circ} \mathrm{C}$ for $30 \mathrm{~s}$, and $72^{\circ} \mathrm{C}$ for $30 \mathrm{~s}$. Reactions were carried out and analyzed using Stratagene MX3005P real-time PCR system (Agilent, Santa Clara, CA). The detection limit of early or late RT DNA products was 10 copies.

\section{Preparation of mouse bone marrow derived macrophages}

Male C57BL/6 mice were sacrificed and the femur and tibia were collected by carefully trimming off muscles and tendons. The bones were rinsed in ice-cold $95 \%$ ethanol for $1 \mathrm{~min}$ and then in PBS. Both ends of the bones were cut off using sterile forceps and scissors. Bone marrow was gently flushed out with RPMI-1640 medium containing 10\% FBS using a $5 \mathrm{~mL}$ syringe and $271 / 2$ gauge needle.

The bone marrow cells were plated at $1 \times 10^{7}$ cells $/ 100 \mathrm{~mm}$ petri dish (bacteriology grade) in $10 \mathrm{~mL}$ RPMI-1640 medium with $10 \%$ FBS and $40 \mathrm{ng} / \mathrm{mL}$ murine M-CSF (Peprotech, Rocky Hill, NJ). The cells were incubated at $37^{\circ} \mathrm{C}$ for 4 days, and then the culture supernatant was removed and replaced with fresh RPMI-1640 medium containing 10\% FBS and $20 \mathrm{ng} / \mathrm{mL}$ murine M-CSF. After differentiation for 5 days, bone marrow derived macrophages (mBMDMs) at $1 \times 10^{5}$ per well in a 48 -well plate were cultured in hormone-free medium for $24 \mathrm{~h}$. Cells were pretreated with or without E2 followed by HIV-1vsv infection.

\section{Statistical analysis}

Differences between data sets were analyzed by a twotailed Student $t$-test and Wilcoxon's signed-rank tests (SAS v9.2; SAS, Inc., Cary, NC). A $p$ value of $<0.05$ was considered significant.

\section{Results}

\section{E2 inhibits HIV-1 infection of primary MDMs}

To determine the effect of sex hormones on HIV infection of primary HIV target cells, activated PBMCs, CD4 + T-cells, or MDMs were pretreated with E2 or P4 at different concentrations for $24 \mathrm{~h}$ before exposure to CCR5 (R5)-using HIV-1 $1_{\text {JR-FL }}$ pseudotyped luciferase reporter virus. E2 or P4 was added back after $2 \mathrm{~h}$ viral exposure, and was present during the infection. E2 exhibited a moderate inhibitory effect on HIV infection of PHA-activated PBMCs and a slight enhancing effect on infection of PHA-activated CD4+ T-cells (Fig. 1 A and B). E2 had no effect on HIV infection of T-cell receptor (TCR)-activated CD4 + T-cells (Fig. 1C), of HeLa-CD4-CCR5 cells, or of THP-1 cell line-derived macrophages (data not shown for HeLa and THP-1 cells). In contrast to the results in PBMCs or CD4 + T-cells, E2 at physiological concentrations $(19,41,48)$, 10 and $100 \mathrm{nM}$, significantly inhibited HIV infection of MDMs (88\% inhibition; Fig. 1D). E2 also blocked HIV replication in MDMs by R5 and dual-tropic X4R5 primary isolates (Fig. 1E). In contrast to E2, P4 at $1-100 \mathrm{nM}$ did not exert any effect on HIV infection of activated PBMCs, CD4 + T-cells, or MDMs.

We further characterized anti-HIV activity of E2 in primary macrophages and found that pretreatment of MDMs with E2 was sufficient to block HIV infection (Fig. 2A). However, prolonged treatment of MDMs with E2 (24 or $48 \mathrm{~h}$ ) but not short-term exposure ( 2 or $8 \mathrm{~h}$ ) was required to block HIV infection (Fig. 2B). Additionally, the anti-HIV activity of E2 was not due to cytotoxicity (Fig. 2C). MDMs were highly sensitive to LPS, a TLR4 agonist, which inhibits HIV infection. To confirm that anti-HIV activity of E2 was not due to LPS contamination, we examined the role of TLR4 activation in E2-mediated HIV inhibition. TLR4 inhibitor (CLI-095) abolished HIV inhibition by LPS but not E2, indicating that the HIV inhibitory effect of E2 was unlikely due to LPS contamination (Fig. 2D).

\section{HIV exposure abolishes E2-mediated HIV inhibition}

We then determined whether E2 exhibited any effect on viral infection in HIV-infected MDMs. Our results indicated that E2 did not have a significant inhibitory effect on HIV infection of MDMs among eight different donors when 
A PHA-activated PBMCs
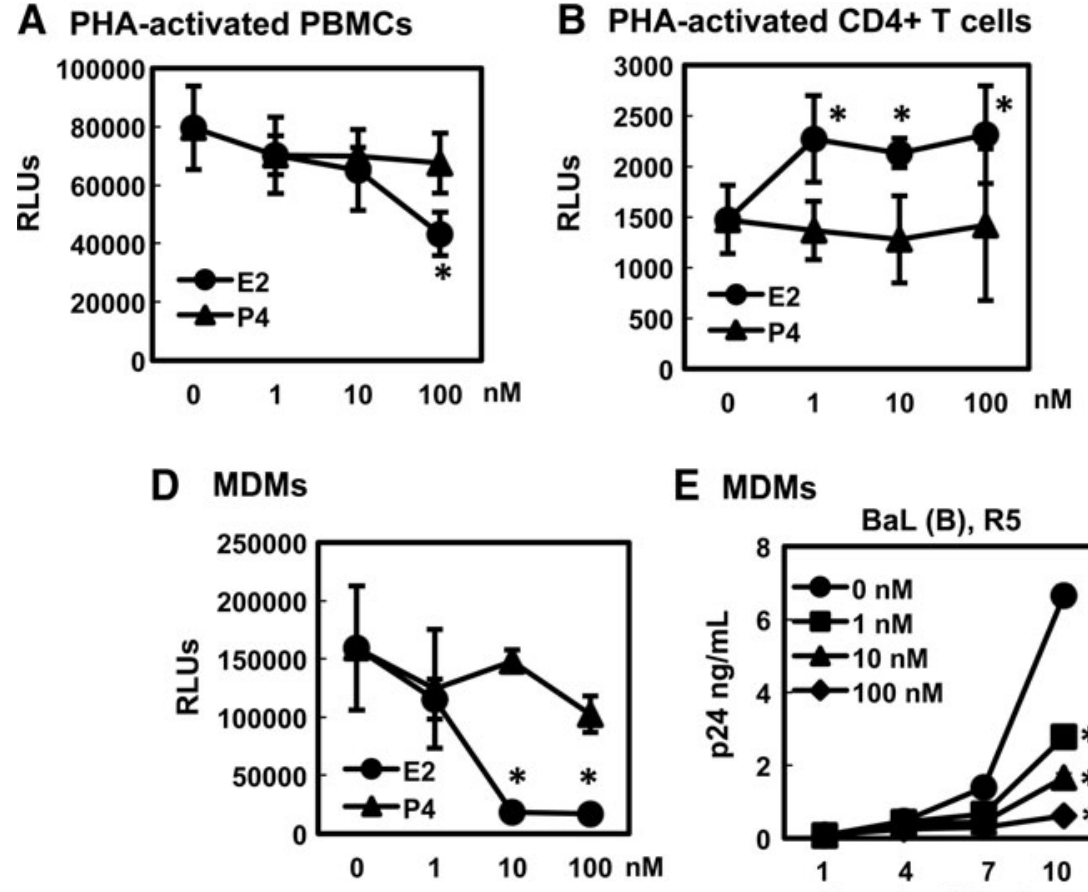

E MDMs

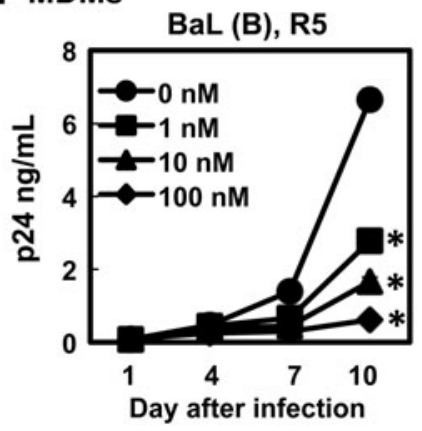

C $\alpha$ CD3/ $\alpha$ CD28-activated CD4+ T cells
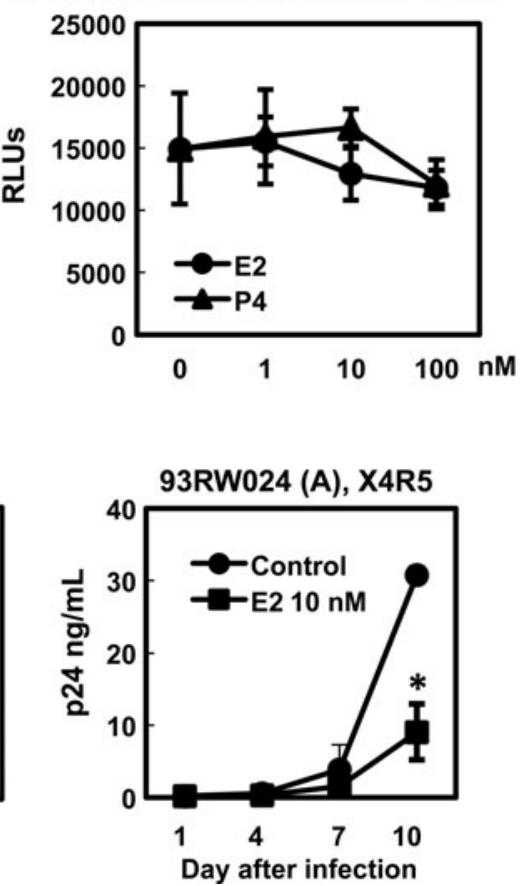

FIG. 1. $17 \beta$-estradiol (E2) inhibits HIV-1 infection of primary monocyte-derived macrophages (MDMs) but not activated peripheral blood mononuclear cells (PBMCs) or CD4 + T-cells. (A)-(D) PHA-activated PBMCs, PHA-activated CD4 + Tcells, anti-CD3 Ab/anti-CD28 Ab-activated CD4 + T-cells, or MDMs were treated with various concentrations of E2 or progesterone (P4) for $24 \mathrm{~h}$ followed by exposure to pseudotyped HIV- $1_{\mathrm{JR}-\mathrm{FL}}$ luciferase reporter virus for $2 \mathrm{~h}$. After washing off unbound virus, E2 or P4 was added back to the culture, and HIV infection was determined by measuring the luciferase activity at day 2-3 after infection. (E) MDMs were treated with E2 at the indicated concentrations for $24 \mathrm{~h}$ followed by exposure to replication competent virus HIV-1 $1_{\mathrm{BaL}}$ or primary isolate 93RW024 (clade A) at a multiplicity of infection (MOI) of 0.05 for $2 \mathrm{~h}$. After washing, E2 was added back to the culture. HIV replication was determined by measuring HIV particles in the culture media at indicated time points using HIV p24 enzyme-linked immunosorbant assay (ELISA). ${ }^{*} p<0.05$, E2-treated MDM versus nontreated controls. Data are presented as mean \pm standard deviation (SD) of triplicate samples and represent three independent experiments.

MDMs were exposed to HIV prior to E2 treatment (Fig. 3A and B). Similar results were obtained when replication competent virus $\mathrm{HIV}-1_{\mathrm{BaL}}$ was used (Fig. 3C).

\section{E2-mediated HIV inhibition occurs after viral entry}

To delineate the specific stages of the HIV life cycle that were blocked by E2, we first assessed whether pretreatment of MDMs with E2 affected HIV attachment. MDMs were treated with different concentrations of $\mathrm{E} 2$ for $24 \mathrm{~h}$ followed by exposure to HIV for $2 \mathrm{~h}$ at $4^{\circ} \mathrm{C}$. After washing, cellassociated HIV p24 was determined by HIV p24 ELISA. There was no difference in HIV attachment in MDMs with or without E2 pretreatment (Fig. 4A). We also found that pretreatment of MDMs with E2 blocked HIV infection by pseudotyped virus with VSV G envelope, which is independent of CD4 and HIV co-receptors for viral entry (Fig. 4B). This result indicated that E2-mediated HIV inhibition was independent of HIV receptors. Additionally, FACS analysis of CD4 and HIV co-receptors demonstrated that E2 did not significantly alter cell surface expression of CD4, CXCR4, or CCR5 (Fig. 4C). Taken together, these results indicate that E2 blocks HIV infection at the postentry level.

We then assessed the effect of E2 on the HIV reverse transcription (RT) step. The kinetics of HIV early and late $\mathrm{RT}$ products in MDMs with or without E2 treatment were determined by quantitative real-time PCR. Early and late RT DNA products in uninfected cells were not detectable (data not shown). In HIV-infected cells, there was no significant difference in the synthesis of early RT products between the control sample (without E2) and E2-treated MDMs (Fig. $5 \mathrm{~A})$. However, the synthesis of late RT products was abolished in E2-treated MDMs (Fig. 5B).

\section{Involvement of IFNs in E2-mediated HIV infection of MDMs}

To examine whether E2 induced a host restriction factor(s) in MDMs that in turn led to HIV inhibition, mRNA expression of IFNs (IFN- $\alpha$, IFN- $\beta$. and IFN- $\gamma$ ) and known HIV host restriction factors (SAMHD1, APOBEC3G, APOBEC3F, and APOBEC3A) $(2,12,20)$ in MDMs with or without E2 treatment was assessed by semi-quantitative real-time RT-PCR analysis. IFN- $\alpha$ and IFN- $\beta$ were significantly induced by E2 in MDMs from several donors, whereas IFN- $\gamma$ was induced only in two out of five donors (Fig. 6). Induction of host restriction factors SAMHD1, APOBEC3G, and APOBEC3F was not consistent in E2treated MDMs. However, the induction of APOBEC3A was consistent among different donors.

IFN- $\alpha$ has been shown to block HIV infection at the step of late $\operatorname{RT}(7,13)$. Because it was consistently induced in 

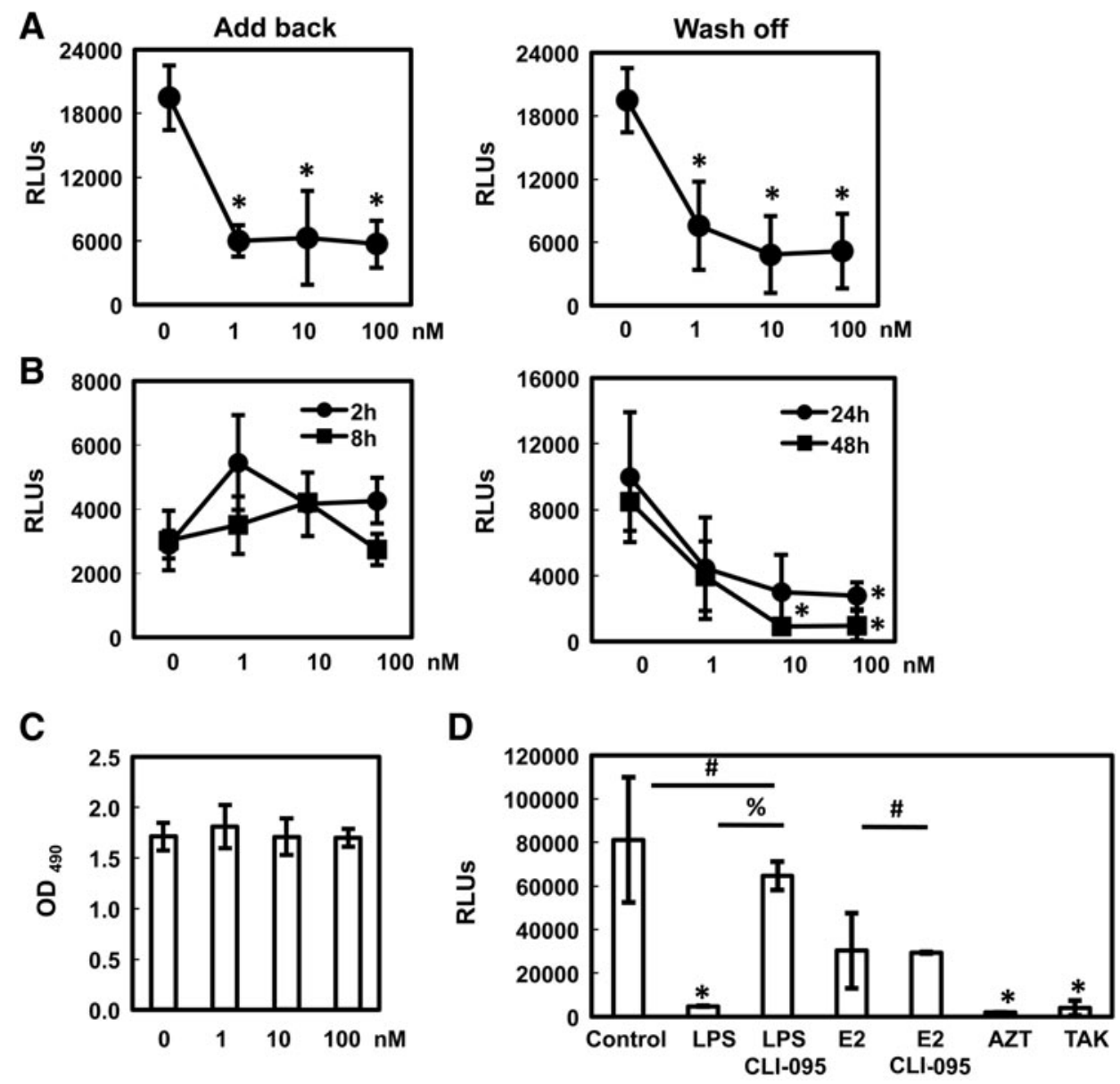

FIG. 2. Prolonged treatment of MDMs with E2 is required to achieve HIV inhibition. (A) MDMs were treated with E2 for $24 \mathrm{~h}$ followed by exposure to HIV-1 $1_{\text {JR-FL }}$ luciferase reporter virus for $2 \mathrm{~h}$. HIV-exposed MDMs were then cultured with E2 (add back) or without E2 (wash off) for 2 days before measurement of luciferase activity. (B) MDMs were treated with E2 at indicated concentrations for $2,8,24$, or $48 \mathrm{~h}$ before HIV infection. In panels (A) and (B), * $p<0.05$, E2-treated MDM versus nontreated controls. (C) MDMs were treated with E2 at indicated concentrations for $24 \mathrm{~h}$ before measurement of cell viability by MTS assay. (D) MDMs were treated with TLR4 inhibitor CLI-095 at $1 \mu \mathrm{g} / \mathrm{mL}$ or $0.1 \%$ DMSO (control) for $2 \mathrm{~h}$ followed by incubation with E2 at $10 \mathrm{nM}$ or LPS at $100 \mathrm{ng} / \mathrm{mL}$ for $24 \mathrm{~h}$. HIV infection was performed as described in Materials and Methods. HIV-1 entry inhibitor TAK779 (TAK, $10 \mu \mathrm{M})$ and reverse transcriptase inhibitor AZT (5 $\mu \mathrm{M})$ were included as a comparison for HIV inhibition. The results shown here are representative of experiments from at least three different donors. ${ }^{*} p<0.05$, E2-, LPS-, AZT-, or TAK-treated MDM versus nontreated MDM; ${ }^{\%} p<0.05$, LPS-treated MDM versus CLI-095/LPS-treated MDM. "\# $p>0.05$ (no significant difference) control MDM versus CLI-095/LPS-treated MDM; E2-treated MDM versus CLI-095/E2-treated MDM. Data are presented as mean \pm SD of triplicate samples.

E2-treated MDMs from many donors, we examined the involvement of IFN- $\alpha$ in E2-mediated HIV inhibition in MDMs. MDMs were treated with E2 in the presence of medium only (no Ab), isotype control Ab, or anti-IFN- $\alpha \mathrm{Ab}$. Anti-IFN- $\alpha \mathrm{Ab}$ abolished the HIV inhibitory effect of E2, whereas E2 blocked HIV infection significantly in MDMs in the presence of medium only or isotype control Ab (Fig. 7A).

The role of IFN- $\alpha$ in E2-mediated HIV inhibition was further confirmed in BMDMs from IFN- $\alpha$ receptor deficient mice $(\operatorname{IFNAR}(-/-))$. Although hCyclin T1 is known to increase HIV RNA transcription significantly in murine cells (51), it is feasible to study HIV gene expression in BMDMs in the absence of hCyclin $\mathrm{T} 1$ by using pseudotyped $\mathrm{HIV}-1_{\text {vsv }}$ luciferase virus to bypass the requirement for HIV entry (35). Wild-type (wt) and $\operatorname{IFNAR}(-/-)$ BMDMs were treated with E2 for $24 \mathrm{~h}$ followed by infection with pseu- dotyped HIV $-1_{\mathrm{vsv}}$ luciferase virus. In agreement with the results using neutralizing Ab, E2 exhibited HIV inhibition in wt BMDMs. However, its HIV inhibitory effect was abolished in IFNAR( $-/-)$ BMDMs (Fig. 7B).

To examine whether the lack of E2-mediated HIV inhibition in HIV-exposed MDMs (Fig. 3) was due to interference with induction of IFNs, MDMs were exposed to HIV-1JR-FL virus for $2 \mathrm{~h}$ before E2 treatment. As a comparison, MDMs were also exposed to virus without HIV Env glycoproteins $(\operatorname{Env}(-))$ virus for $2 \mathrm{~h}$ followed by E2 treatment. Gene expression of IFNs was determined by semiquantitative RT-PCR analysis. We found that E2-mediated induction of IFNs was partially reduced (by 37\%) when MDMs were exposed to $\operatorname{Env}(-)$ virus. However, exposure to HIV-1JR-FL completely abolished induction of IFNs by E2 (Fig. 7C). 
A
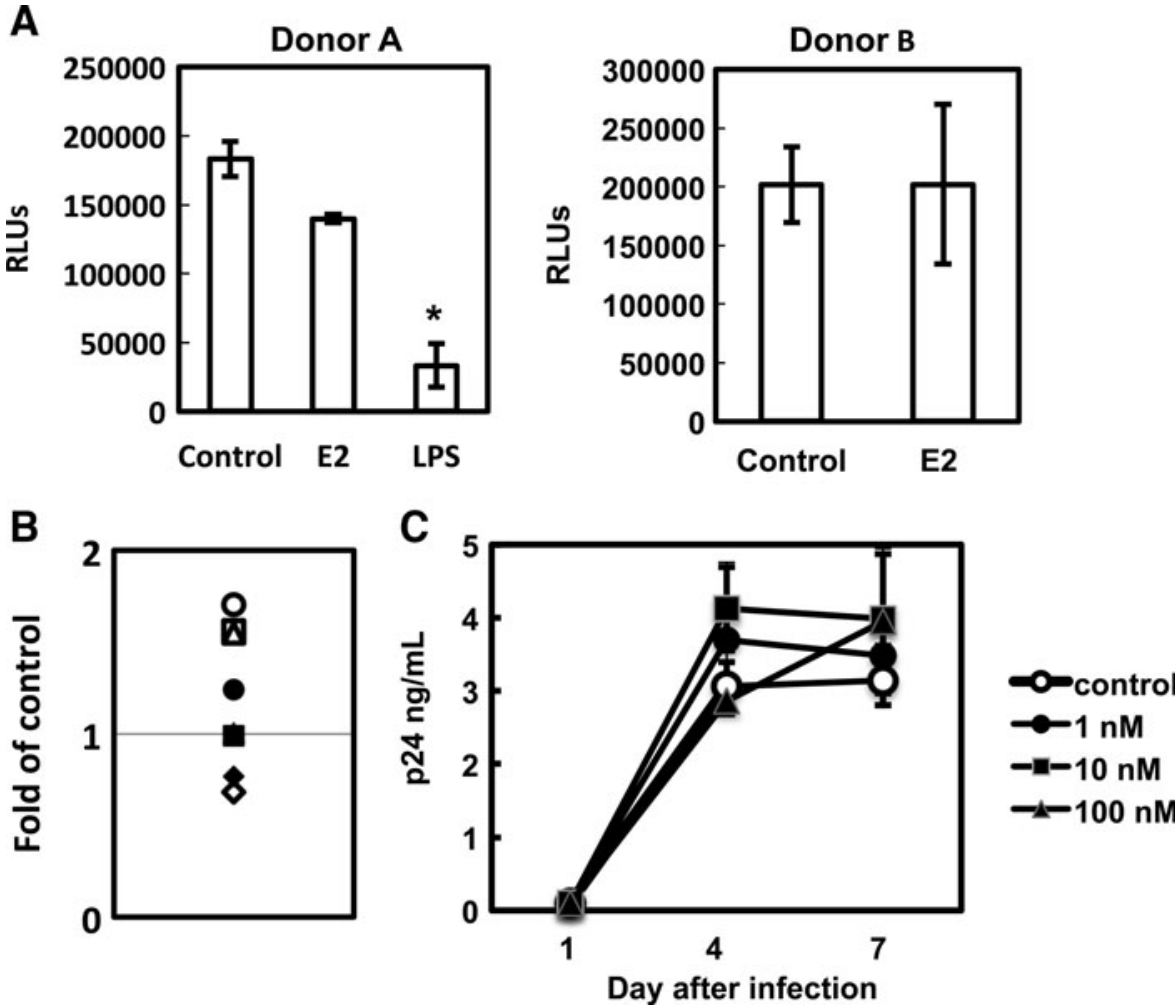

FIG. 3. E2 does not inhibit HIV infection in HIV-infected MDMs. (A) MDMs were exposed to pseudotyped HIV-1 ${ }_{\mathrm{JR}}$-FL luciferase reporter virus for $2 \mathrm{~h}$ before incubation with or without $\mathrm{E} 2$ at $10 \mathrm{nM}$ for 2 days before measuring luciferase activity. Data represent two experiments from two of eight donors. LPS was included as a control. There was no difference in HIV infection in control or E2-treated samples when macrophages were exposed to HIV before E2 treatment. $* p<0.05$, LPS-treated MDM versus nontreated controls. Data are mean \pm SD of triplicate samples. (B) Summary of the effect of E2 on HIV-1 infection of MDMs from eight donors when E2 was added after cells were exposed to HIV. The data are presented as fold of control, calculated as the fold of HIV infection (relative light units) in E2-treated MDMs compared to untreated controls. (C) MDMs were exposed to HIV-1 $1_{\mathrm{BaL}}$ for $2 \mathrm{~h}$, washed, and then cultured in the presence of E2 at different concentrations. Infected cells without E2 treatment were included as a control. There was no difference in HIV infection between E2-treated versus nontreated controls.

\section{Discussion}

The immunoregulatory functions of estrogen are well documented (reviewed in $(19,46))$. However, the protective role of estrogen in HIV/SIV transmission has been primarily attributed to an increase in thickness of the vaginal wall (19). A recent report demonstrates E2 inhibits $\mathrm{HIV}-1_{\mathrm{BaL}}$ replication in activated PBMCs by $50 \%$ (47). We observed a moderate inhibitory effect in activated PBMCs but not in activated CD4 + T-cells using a single-cycle infection assay (Fig. 1A-C). However, our results demonstrate that macrophages are the main target for the anti-HIV effect of estrogen. Pretreatment with E2 blocked the early phase of HIV infection of primary macrophages. E2 induced gene expression of type I IFNs and myeloid cell-specific host restriction factor APOBEC3A. We also demonstrated that IFN- $\alpha$ contributes to E2-mediated HIV inhibition.

HIV restriction by IFN- $\alpha$ has been shown to occur at the step of RT in primary macrophages, and the inhibitory effect is more pronounced on the synthesis of late RT products than on synthesis of early RT products $(1,7,13)$. IFN- $\alpha$ preferentially inhibits HIV replication in MDMs compared to CD4 + T-cells $(1,7,13)$. Our results show similar profiles of HIV restriction in E2-treated macrophages. E2-mediated HIV in- hibition was found primarily in primary macrophages and occurred at the step of late RT. Our studies using neutralizing $\mathrm{Ab}$ against IFN- $\alpha$ and IFNAR $(-/-)$ mice indicated that IFN$\alpha$ played a major role in E2-mediated HIV inhibition in macrophages. IFN- $\alpha$ is known to induce HIV host restriction factors such as APOBEC family members and SAMHD1, which can act on early steps in the HIV life cycle (reviewed in $(29,54)$ ). Although the contribution of APOBEC3G to IFN$\alpha$-mediated HIV inhibition in MDMs has not been consistent $(7,29,39)$, we did not observe induction of most host restriction factors (except APOBEC3A) in E2-treated MDMs among different donors. We speculate that the lack of consistent induction of APOBEC3G, APOBEC3F, or SAMHD1 in E2-treated MDMs may be because E2 treatment induces lower concentrations of IFN- $\alpha$ than are commonly used for exogenous stimulation $(10 \mathrm{ng} / \mathrm{mL})$. Interestingly, induction of APOBEC3A was associated with IFN- $\alpha$ induction and was consistently observed in E2-treated MDM from several donors (Fig. 6). APOBEC3A can be induced by IFN- $\alpha$ and inhibits ethe early phase of HIV infection in cells of myeloid origins (2). Thus, APOBEC3A may in part contribute to E2mediated HIV inhibition through IFN- $\alpha$ induction.

Estrogen receptor (ER) signaling is activated through the binding of estrogen to ER- $\alpha$ or ER- $\beta$, and it has been 
A

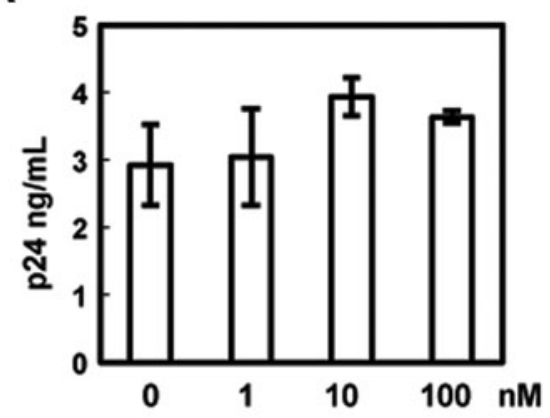

B

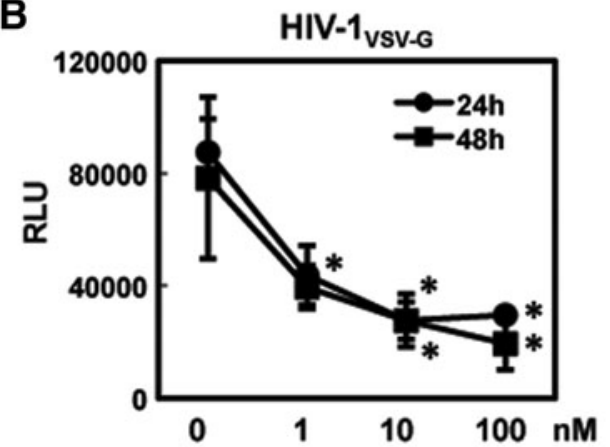

C
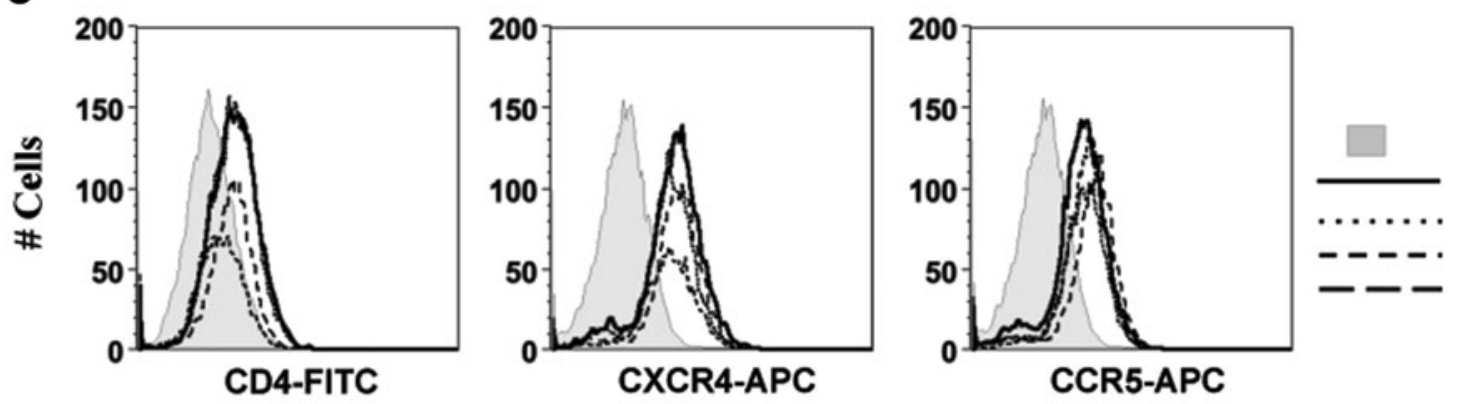

Isotype control

Medium

E2 $1 \mathrm{nM}$

E2 $10 \mathrm{nM}$

E2 $100 \mathrm{nM}$
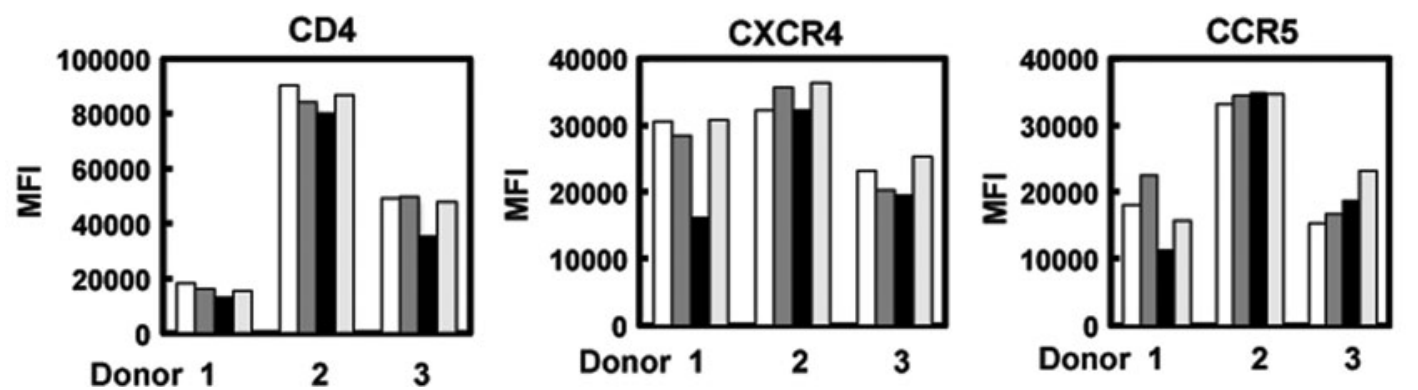

口control
पE2 $1 \mathrm{nM}$
-E2 10nM

口E2 100nM

FIG. 4. E2 does not inhibit the steps of HIV attachment and viral entry. (A) MDMs were treated with the indicated concentrations of E2 for $24 \mathrm{~h}$ followed by exposure to pseudotyped HIV-1 JR-FL luciferase reporter virus at $4{ }^{\circ} \mathrm{C}$ for $2 \mathrm{~h}$. MDMs were washed four times with cold phosphate-buffered saline (PBS) and then lysed in 1\% Triton X-100. The level of cell-associated HIV-1 p24 proteins was determined by p24 ELISA. The difference in the level of HIV p24 proteins between E2-treated and nontreated MDMs was not significant. (B) MDMs were treated with the indicated concentrations of E2 for 24 or $48 \mathrm{~h}$ before infection with pseudotyped HIV-1 VSV-G luciferase reporter virus (10 ng of HIVp24 per sample). HIV infection was determined by measuring luciferase activity at day 2 after infection. $* p<0.05$, E2-treated MDM versus nontreated controls. Data are mean \pm SD of triplicate samples and represent two independent experiments. (C) Cell surface expression of CD4, CXCR4, and CCR5 on MDMs with or without treatment with the indicated concentrations of E2 for $24 \mathrm{~h}$ was determined by FACS analysis. Mean fluorescence intensity (MFI) of cell surface CD4, CXCR4, and CCR5 in control and E2-treated MDMs from three donors is shown.

established that cross-talk between ER signaling and the $\mathrm{NF}-\kappa \mathrm{B}$ pathway suppresses NF- $\kappa \mathrm{B}$-mediated inflammation $(3,5)$. While the molecular mechanism of the interaction between the ER pathway and IFN signaling is not well characterized, the impact of estrogen on IFN-mediated immune responses is evident. For example, E2 suppresses IFN- $\gamma$ mediated interferon regulatory factor I (IRF-1) induction in murine lymphocytes (25), and suppresses IFN- $\gamma$-mediated interferon-induced protein of $10 \mathrm{kDa}$ (IP10) induction in human keratinocytes (22). Additionally, pretreatment of monocyte-derived dendritic cells blocks type I IFN production induced by RNA viruses (11). In contrast to suppression of IFN-mediated gene induction, E2 increases IFN- $\gamma$ production by activated lymphocytes from E2-treated ovariec- tomized mice (23). In this study, we demonstrated that E2 induces type I IFNs, and that IFN- $\alpha$ contributes to HIV restriction in human MDMs. Similarly, E2 induces production of IFN- $\alpha$ by murine plasmacytoid dendritic cells (pDCs), the major IFN- $\alpha$ producing immune cells, and enhances the levels of IFN- $\alpha$ inpDCs in response to CpG, a TLR9 agonist, which modulates immune responses by pDCs (26). Although the effect of E2 on IFN responses may depend on the specific target cell and the experimental system, understanding the molecular mechanism of the interaction between ER and IFN pathways may allow us to develop specific anti-HIV inhibitors that will avoid pleiotropic effects of E2 and IFN- $\alpha$.

In contrast to TLR4-mediated suppression of HIV proviral DNA activation through IFN- $\beta$ in MDMs (27), E2 did 
A
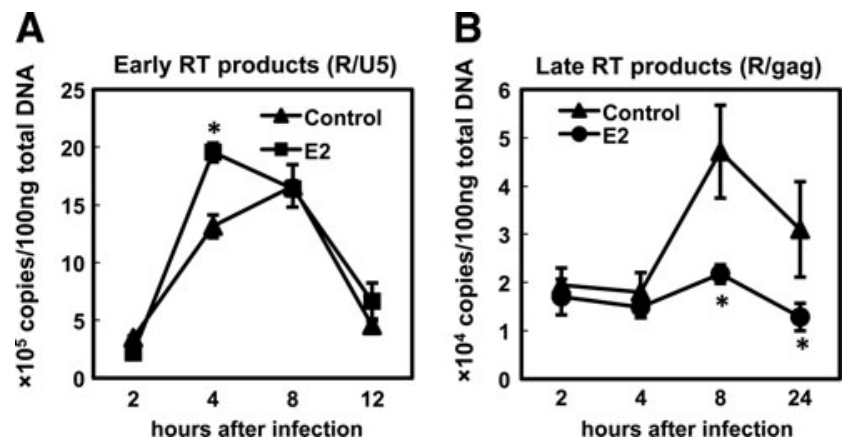

FIG. 5. E2 blocks HIV-1 infection of MDMs at the step of late reverse transcription (RT). MDMs were treated with $10 \mathrm{nM}$ E2 for $24 \mathrm{~h}$ followed by infection with pseudotyped

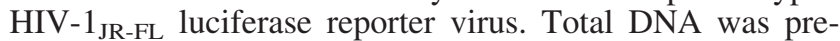
pared at different time points after infection, and HIV-1 RT products were determined by quantitative real-time polymerase chain reaction (PCR) using primer sets specific for early RT products (A) or late RT products $(\mathbf{B}) .{ }^{*} p<0.05$, E2-treated MDM versus nontreated controls. Data are mean \pm SD of triplicate samples and are representative of three independent experiments.

not exert a significant inhibitory effect when MDMs were exposed to HIV prior to E2 treatment. Since we later discovered that E2-mediated HIV restriction occurs at the step of RT, HIV may have completed the step of RT before E2 induced sufficient amounts of IFNs to suppress HIV infection. Additionally, HIV may interfere with E2-mediated IFN induction, resulting in the lack of protection in MDMs with infection by replication competent viruses (Fig. 3C). To test this possibility, we examined the effect of E2 on IFN production in HIV-exposed MDMs, and found that induction of IFNs was abolished in HIV-exposed MDMs (Fig. 7C). We also found that HIV Env(-) virus particles also exerted a moderate inhibitory effect on E2-mediated IFN induction. The molecular mechanism underlying the interference of HIV in innate immune responses appears to be cell-type specific. HIV causes significant IRF3 degradation in transformed T-cells, although the degree of IRF3 degradation is weak in PBMCs (10). HIV accessory proteins Vpr and Vif (37) as well as Vpu (9) have been shown to be involved in IRF3 degradation. In MDDCs, the blockade of IRF3 activation rather than IRF3 degradation contributes to HIVmediated IFN inhibition (16). Additionally, HIV Vpr but not Vif contributes to the inhibitory effect (16). Suppression of type I IFN-stimulated genes by HIV has recently been reported in primary macrophages, although the mechanism is not clear (52). We speculate that the mechanism of HIVmediated IFN inhibition in MDMs may not be the same as in transformed cells or MDDCs because HIV particles without Env were able to suppress IFN production. The detailed mechanism is currently under investigation.

Finally, we note that our results conflict with a recent report indicating that E2 partially inhibits HIV infection of both activated CD4 + T-cells and primary macrophages (41). However, in contrast to our results, pretreatment of cells with E2 did not block pseudotyped HIV-1 ${ }_{\mathrm{vsv}}$ virus. Thus, the authors concluded that E2 blocks the step of viral attachment, since expression of CCR5 was not altered in E2treated cells. It is likely that differences in preparation of primary cells and the protocol of E2 treatment produced this discrepancy. We pretreated fully activated CD4+ T-cells
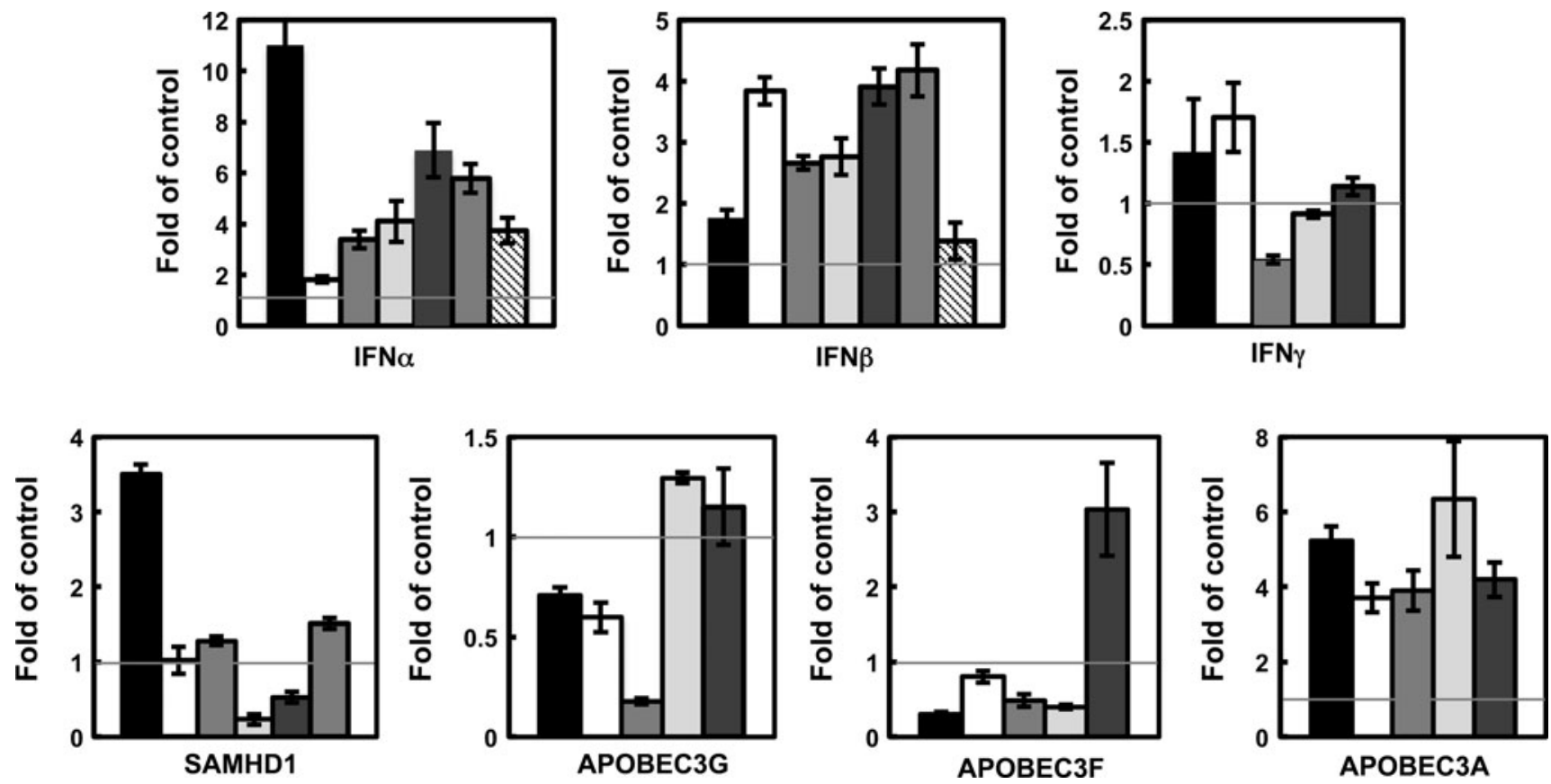

FIG. 6. E2 induces expression of interferon-alpha (IFN- $\alpha)$ and APOBEC3A in MDMs from different donors. MDMs were treated with E2 at $10 \mathrm{nM}$ for $24 \mathrm{~h}$. Total RNA was prepared, and gene expression of IFNs or specific host restriction factors was measured by semi-quantitative real-time RT-PCR. Fold of control for each gene was calculated based on $\Delta \Delta C t$ as described in Materials and Methods. The bars in each panel represent the fold changes in E2-treated MDMs from different donors. The gray line indicates no changes in the level of gene expression between treated and untreated samples. 
A

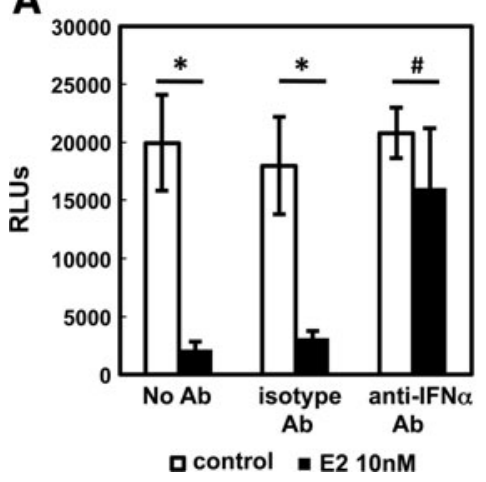

B

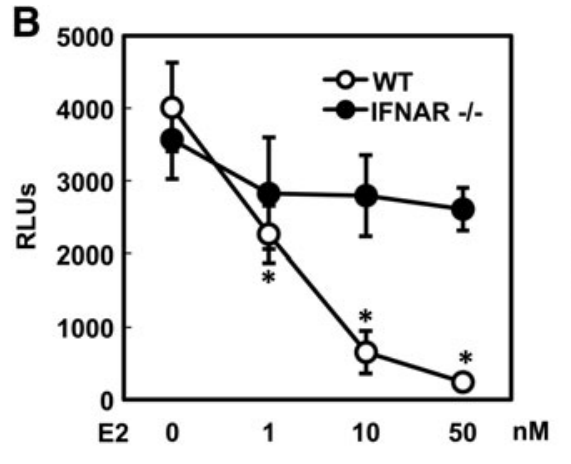

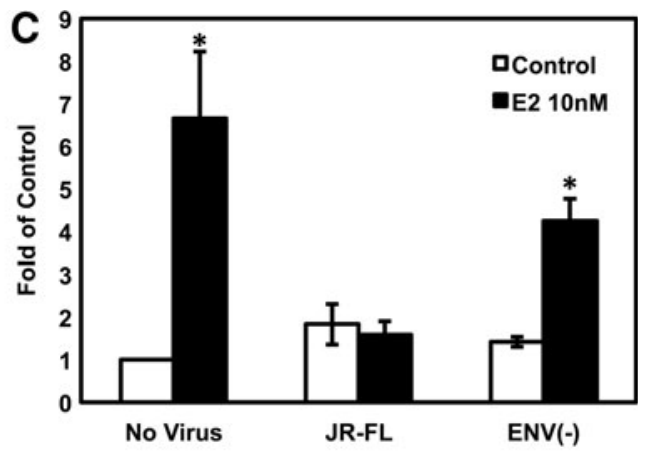

FIG. 7. IFN- $\alpha$ contributes to E2-mediated HIV inhibition in MDMs. (A) MDMs were treated with E2 at $10 \mathrm{nM}$ in the presence of medium only, isotype control Ab, or anti-IFN- $\alpha$ Ab at $10 \mu \mathrm{g} / \mathrm{mL}$ for $24 \mathrm{~h}$. MDMs were then infected with pseudotyped HIV-1 $1_{\text {JR-FL }}$ luciferase reporter virus for $2 \mathrm{~h}$. After washing off unbound virus, E2 and antibodies (control Ab or anti-IFN- $\alpha \mathrm{Ab}$ ) were added back and were present during infection. HIV infection was determined by measuring luciferase activity at day 2 after infection. (B) Bone marrow derived macrophages (BMDMs) from C57BL/6 WT mice or IF$\operatorname{NAR}(-/-)$ mice were treated with E2 at the indicated concentrations for $24 \mathrm{~h}$. BMDMs were infected with pseudotyped HIV-1 VsV-G luciferase reporter virus in the presence of E2 for 2 days. HIV infection was determined by measuring luciferase activity at day 2 after infection. $* p<0.05$, E2-treated samples versus nontreated controls in both no Ab- and isotype Ab-treated samples. \# $\mathrm{p}>0.05$ (no significant difference), E2-treated samples versus nontreated controls in anti-IFN$\alpha \mathrm{Ab}$-treated samples. Data are mean $\pm S D$ of triplicate samples and are representative of two independent experiments. (C) MDMs were exposed to HIV-1 $1_{\text {JR-FL }}$ or to virus particles without Env $(\operatorname{HIV} \operatorname{Env}(-))$ for $2 \mathrm{~h}$ before treatment with or without $\mathrm{E} 2$ at $10 \mathrm{nM}$ for $6 \mathrm{~h}$. Uninfected MDMs were included as a control. Total RNA was prepared and gene expression of type I IFNs was analyzed by quantitative real-time PCR. Result for IFN $\alpha$ expression are shown. $* p<0.025$, E2-treated samples versus nontreated controls in no virus and HIV-1 ENV( - ) samples. Data are mean $\pm S D$ of triplicate samples. Similar results were observed in MDMs from two donors.

with E2 versus pretreatment of CD4+ T-cells with E2 during activation (41). We treated MDMs with E2 at day 7 after differentiation, at which time the majority of MDMs expressed CD68, a differentiation marker for mature macrophages, versus treatment of MDMs with E2 at day 4 of differentiation, prior to maturation (41).

In summary, our study offers a new mechanism by which E2 protects against HIV infection through induction of IFNs. Further investigation on the interplay between HIV and innate immune responses regulated by E2 will be important for development of better strategies for HIV prevention.

\section{Acknowledgments}

We thank George Yap and Yungtai Lo for their help on preparation of mBMDMs and on statistical analysis, respectively. This work is supported by NIH grants AI093196, AI081559, and AI110372 (to T.L.C.). C.T. was a recipient of the Conference on Retroviruses and Opportunistic Infections (CROI) Young Investigator Award.

\section{Author Disclosure Statement}

No competing financial interests exist.

\section{References}

1. Baca-Regen L, Heinzinger N, Stevenson M, and Gendelman HE. Alpha interferon-induced antiretroviral activities: restriction of viral nucleic acid synthesis and progeny virion production in human immunodeficiency virus type 1-infected monocytes. J Virol 1994;68:7559-7565.
2. Berger G, Durand S, Fargier G, et al. APOBEC3A is a specific inhibitor of the early phases of HIV-1 infection in myeloid cells. PLoS Pathog 2011;7:e1002221.

3. Biswas DK, Singh S, Shi Q, Pardee AB, and Iglehart JD. Crossroads of estrogen receptor and NF-kappaB signaling. Sci STKE 2005;pe27.

4. Calippe B, Douin-Echinard V, Delpy L, et al. 17Beta-estradiol promotes TLR4-triggered proinflammatory mediator production through direct estrogen receptor alpha signaling in macrophages in vivo. J Immunol 2010;185:1169-1176.

5. Chadwick CC, Chippari S, Matelan E, et al. Identification of pathway-selective estrogen receptor ligands that inhibit NF-kappaB transcriptional activity. Proc Natl Acad Sci U S A 2005;102:2543-2548.

6. Chang TL, Vargas J Jr, DelPortillo A, and Klotman ME. Dual role of alpha-defensin-1 in anti-HIV-1 innate immunity. J Clin Invest 2005;115:765-773.

7. Cheney KM, and McKnight A. Interferon-alpha mediates restriction of human immunodeficiency virus type-1 replication in primary human macrophages at an early stage of replication. PLoS One 2010;5:e13521.

8. Connor RI, Sheridan KE, Ceradini D, Choe S, and Landau NR. Change in coreceptor use coreceptor use correlates with disease progression in HIV-1-infected individuals. J Exp Med 1997;185:621-628.

9. Doehle BP, Chang K, Rustagi A, McNevin J, McElrath MJ, and Gale M Jr. Vpu mediates depletion of interferon regulatory factor 3 during HIV infection by a lysosomedependent mechanism. J Virol 2012;86:8367-8374.

10. Doehle BP, Hladik F, McNevin JP, McElrath MJ, and Gale M Jr. Human immunodeficiency virus type 1 mediates global disruption of innate antiviral signaling and immune defenses within infected cells. J Virol 2009;83:10395-10405. 
11. Escribese MM, Kraus T, Rhee E, Fernandez-Sesma A, Lopez CB, and Moran TM. Estrogen inhibits dendritic cell maturation to RNA viruses. Blood 2008;112:4574-4584.

12. Goila-Gaur R, and Strebel K. HIV-1 Vif, APOBEC, and intrinsic immunity. Retrovirology 2008;5:51.

13. Goujon C, and Malim MH. Characterization of the alpha interferon-induced postentry block to HIV-1 infection in primary human macrophages and T cells. J Virol 2010;84: 9254-9266.

14. Gupta S, Kumar N, Singhal N, Manektala U, Jain S, and Sodhani P. Cytohormonal and morphological alterations in cervicovaginal smears of postmenopausal women on hormone replacement therapy. Diagn Cytopathol 2006;34: 676-681.

15. Harkonen PL, and Vaananen HK. Monocyte-macrophage system as a target for estrogen and selective estrogen receptor modulators. Ann N Y Acad Sci 2006;1089:218-227.

16. Harman AN, Lai J, Turville S, et al. HIV infection of dendritic cells subverts the IFN induction pathway via IRF1 and inhibits type 1 IFN production. Blood 2011;118: 298-308.

17. Heffron R, Donnell D, Rees H, et al. Use of hormonal contraceptives and risk of HIV-1 transmission: a prospective cohort study. Lancet Infect Dis 2012;12:19-26.

18. Heffron R, Mugo N, Ngure K, et al. Hormonal contraceptive use and risk of HIV-1 disease progression. Aids 2013; 27:261-267.

19. Hel Z, Stringer E, and Mestecky J. Sex steroid hormones, hormonal contraception, and the immunobiology of human immunodeficiency virus-1 infection. Endocr Rev 2010;31: 79-97.

20. Hrecka K, Hao C, Gierszewska M, et al. Vpx relieves inhibition of HIV-1 infection of macrophages mediated by the SAMHD1 protein. Nature 2011;474:658-661.

21. Hubacher D, Mavranezouli I, and McGinn E. Unintended pregnancy in sub-Saharan Africa: magnitude of the problem and potential role of contraceptive implants to alleviate it. Contraception 2008;78:73-78.

22. Kanda N, and Watanabe S. 17beta-estradiol inhibits the production of interferon-induced protein of $10 \mathrm{kDa}$ by human keratinocytes. J Invest Dermatol 2003;120:411-419.

23. Karpuzoglu-Sahin E, Hissong BD, and Ansar Ahmed S. Interferon-gamma levels are upregulated by 17-betaestradiol and diethylstilbestrol. J Reprod Immunol 2001;52: 113-127.

24. Kramer PR, Kramer SF, and Guan G. 17 beta-estradiol regulates cytokine release through modulation of CD16 expression in monocytes and monocyte-derived macrophages. Arthritis Rheum 2004;50:1967-1975.

25. Lengi AJ, Phillips RA, Karpuzoglu E, and Ahmed SA. Estrogen selectively regulates chemokines in murine splenocytes. J Leukoc Biol 2007;81:1065-1074.

26. Li X, Xu Y, Ma L, Sun L, Fu G, and Hou Y. 17betaestradiol enhances the response of plasmacytoid dendritic cell to CpG. PLoS One 20009;4:e8412.

27. Liu X, Mosoian A, Li-Yun Chang T, et al. Gonococcal lipooligosaccharide suppresses HIV infection in human primary macrophages through induction of innate immunity. J Infect Dis 2006;194:751-759.

28. Lutalo T, Musoke R, Polis C, et al. Effects of hormonal contraceptive use on HIV acquisition in women and transmission to men among HIV-discordant couples, Rakai, Uganda, Abstract \#563, 19th Conference on Retroviruses and Opportunistic Infections, Seattle, WA, 2012.
29. Malim MH, and Bieniasz PD. HIV Restriction Factors and Mechanisms of Evasion. Cold Spring Harb Perspect Med 2012;2:a006940.

30. Marx PA, Spira AI, Gettie A, et al. Progesterone implants enhance SIV vaginal transmission and early virus load. Nat Med 1996;2:1084-1089.

31. McCoy S, Zheng W, Montgomery ET, et al. Oral and injectable contraception use and risk of HIV acquisition among women: MIRA study, p. 20LB, 19th Conference on Retroviruses and Opportunistic Infections, Seattle, WA, 2012.

32. Messingham KA, Heinrich SA, and Kovacs EJ. Estrogen restores cellular immunity in injured male mice via suppression of interleukin-6 production. J Leukoc Biol 2001; 70:887-895.

33. Molander U, Milsom I, Ekelund P, Mellstrom D, and Eriksson O. Effect of oral oestriol on vaginal flora and cytology and urogenital symptoms in the post-menopause. Maturitas 1990;12:113-120.

34. Morrison CS, Skoler-Karpoff S, Kwok C. Hormonal contraception and the risk of HIV acquisition among women in South Africa. Aids 2012;26:497-504.

35. Mosoian A, Teixeira A, Burns CS, et al. Prothymosin-alpha inhibits HIV-1 via Toll-like receptor 4-mediated type I interferon induction. Proc Natl Acad Sci U S A 2010;107: 10178-10183.

36. Nilsson K, Risberg B, and Heimer G. The vaginal epithelium in the postmenopause - cytology, histology and $\mathrm{pH}$ as methods of assessment. Maturitas 1995;21:51-56.

37. Okumura A, Alce T, Lubyova B, Ezelle H, Strebel K, and Pitha PM. HIV-1 accessory proteins VPR and Vif modulate antiviral response by targeting IRF-3 for degradation. Virology 2008;373:85-97.

38. Pask AJ, McInnes KJ, Webb DR, and Short RV. Topical oestrogen keratinises the human foreskin and may help prevent HIV infection. PLoS One 2008;3:e2308.

39. Peng G, Lei KJ, Jin W, Greenwell-Wild T, and Wahl SM. Induction of APOBEC3 family proteins, a defensive maneuver underlying interferon-induced anti-HIV-1 activity. J Exp Med 2006;203:41-46.

40. Plummer FA, Simonsen JN, Cameron DW, et al. Cofactors in male-female sexual transmission of human immunodeficiency virus type 1. J Infect Dis 1991;163: 233-239.

41. Rodriguez-Garcia M, Biswas N, Patel MV, et al. Estradiol reduces susceptibility of CD4 + T cells and macrophages to HIV-infection. PLoS One 2013;8:e62069.

42. Rollenhagen C, and Asin SN. Enhanced HIV-1 replication in ex vivo ectocervical tissues from post-menopausal women correlates with increased inflammatory responses. Mucosal Immunol 2011;4:671-681.

43. Shen R, Richter HE, and Smith PD. Early HIV-1 target cells in human vaginal and ectocervical mucosa. Am J Reprod Immunol 2011;65:261-267.

44. Sikoski P, Register TC, Lees CJ, et al. Effects of two novel selective estrogen receptor modulators, raloxifene, tamoxifen, and ethinyl estradiol on the uterus, vagina and breast in ovariectomized cynomolgus monkeys (Macaca fascicularis). Am J Obstet Gynecol 2007;196:75 e71-77.

45. Smith SM, Baskin GB, and Marx PA. Estrogen protects against vaginal transmission of simian immunodeficiency virus. J Infect Dis 2000;182:708-715.

46. Straub RH. The complex role of estrogens in inflammation. Endocr Rev 2007;28:521-574. 
47. Szotek EL, Narasipura SD, and Al-Harthi L. 17beta-Estradiol inhibits HIV-1 by inducing a complex formation between beta-catenin and estrogen receptor alpha on the HIV promoter to suppress HIV transcription. Virology 2013;443: 375-383.

48. Tourgeman DE, Gentzchein E, Stanczyk FZ, and Paulson RJ. Serum and tissue hormone levels of vaginally and orally administered estradiol. Am J Obstet Gynecol 1999;180: 1480-1483.

49. Trunova N, Tsai L, Tung S, et al. Progestin-based contraceptive suppresses cellular immune responses in SHIVinfected rhesus macaques. Virology 2006;352:169-177.

50. Wand H, and Ramjee G. The effects of injectable hormonal contraceptives on HIV seroconversion and on sexually transmitted infections. Aids 2012;26:375-380.

51. Wei P, Garber ME, Fang SM,. Fischer WH, and Jones KA. A novel CDK9-associated C-type cyclin interacts directly with HIV-1 Tat and mediates its high-affinity, loop-specific binding to TAR RNA. Cell 1998;92:451-462.
52. Wie $\mathrm{SH}, \mathrm{Du} \mathrm{P}$, Luong $\mathrm{TQ}$, et al. HIV Downregulates Interferon-Stimulated Genes in Primary Macrophages. J Interferon Cytokine Res 2013;33:90-95.

53. Zack JA, Arrigo SJ, Weitsman SR, et al. HIV-1 entry into quiescent primary lymphocytes: molecular analysis reveals a labile, latent viral structure. Cell 1990;61:213-222.

54. Zheng YH, Jeang KT, and Tokunaga K. Host restriction factors in retroviral infection: promises in virus-host interaction. Retrovirology 2012;9:112.

Address correspondence to: Dr. Theresa L. Chang Public Health Research Institute RBHS-New Jersey Medical School 225 Warren Street Newark, NJ 07103

E-mail: changth@njms.rutgers.edu 